\title{
Published as:
}

Mthokozisi Sibanda, Walter Focke, Leo Braack, Andreas Leuteritz, Harald Brünig, Nguyen Hoai An Tran, Florian Wieczorek, Wolfgang Trümper. Bicomponent fibres for controlled release of volatile mosquito repellents. Materials Science and Engineering: C 91 (2018) 754-761. https://doi.org/10.1016/j.msec.2018.06.016

\section{Bicomponent fibres for controlled release of volatile mosquito repellents}

Mthokozisi Sibanda ${ }^{\mathrm{a}, \mathrm{b}}$, Walter Focke ${ }^{\mathrm{a}, \mathrm{b}, *}$, Leo Braack ${ }^{\mathrm{a}}$, Andreas Leuteritz ${ }^{\mathrm{c}}$, Harald Brünig ${ }^{\mathrm{c}}$, Nguyen Hoai An Tran ${ }^{\mathrm{c}, \mathrm{d}}$, Florian Wieczorek ${ }^{\mathrm{d}}$, and Wolfgang Trümper ${ }^{\mathrm{d}}$

${ }^{a}$ University of Pretoria Institute for Sustainable Malaria Control and MRC Collaborating Centre for malaria research, University of Pretoria, Private Bag X323, Pretoria 0001

${ }^{\text {b} I n s t i t u t e ~ o f ~ A p p l i e d ~ M a t e r i a l s, ~ D e p a r t m e n t ~ o f ~ C h e m i c a l ~ E n g i n e e r i n g, ~ U n i v e r s i t y ~ o f ~ P r e t o r i a, ~ P r i v a t e ~ B a g ~ X 20, ~}$ Hatfield 0028

cLeibniz Institute of Polymer Research Dresden (IPF), Hohe Straße 6, 01069 Dresden

dTechnische Universität Dresden, Fakultät Maschinenwesen, Institut für Textilmaschinen und Textile

Hochleistungswerkstofftechnik (ITM), 01062 Dresden

*Corresponding author: walter.focke@up.ac.za

\section{Highlights}

- Bicomponent fibres prepared by a thermoplastic melt spinning process

- Repellent-plasticised core polymer (EVA) acts as reservoir for the mosquito repellent.

- Membrane-like HDPE sheath controls the release rate, reducing the rate of evaporation.

- Knitted socks showed excellent mosquito repellence for several months.

\begin{abstract}
Core-sheath structured fibres were developed for application as part of an alternative malaria vector control intervention aimed at reducing outdoor malaria transmission. The fibres were prepared by melt spinning of high density polyethylene (HDPE) as sheath and with a concentrate containing volatile N,N-Diethyl-m-toluamide (DEET) in poly(ethylene-co-vinyl acetate) (EVA) as core. The concentrate was prepared by a simple absorption processes to a content up to $40 \mathrm{wt} .-\%$ DEET. Scanning electron microscope imaging confirmed the formation of a bicomponent core-sheath fibre structure. Confocal Raman spectroscopy revealed the development of a concentration gradient of DEET in the sheath layer, suggesting a diffusion controlled release process. Excellent processability was demonstrated on an extrusion system melt spinning with take up speeds reaching $3000 \mathrm{~m} \mathrm{~min}^{-1}$. Sample textiles knitted from such filaments showed high residual repellence activity even after 20 cold washes or after eight months ageing under laboratory conditions. These findings indicate that this technology offers an alternative way to prevent outdoor mosquito bites in an effective and affordable manner.
\end{abstract}

Key words: malaria; mosquitoes; repellent; controlled release; bicomponent fibre 


\section{Graphical abstract}

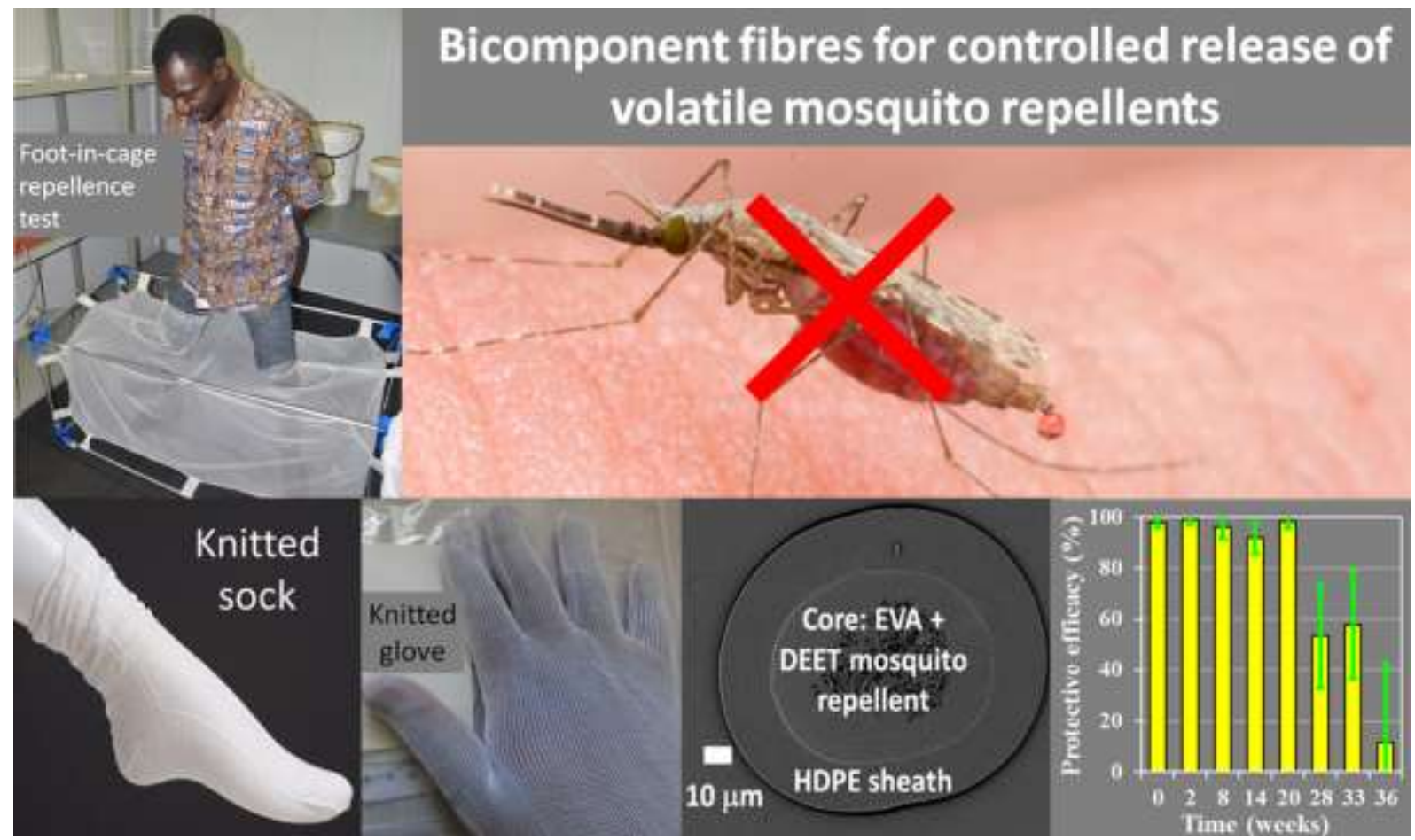

\section{Introduction}

Mosquito-borne pathogens remain the single most important cause of infectious disease on our planet. Of these, malaria tops the list, with 216 million cases recorded globally in 2016, resulting in 445,000 deaths [1]. Lymphatic filariasis, also known as elephantiasis which potentially manifests as painful and profoundly disfiguring cases, afflicted an estimated 120 million people according to a 2007 report [2]. Increasing concern is also widely being expressed due to the spread of a number of mosquito-borne arboviruses across large regions of the globe, including dengue, chikungunya, Zika, but also several others affecting millions of people $[3,4]$. Control of these diseases is in most cases based on interventions aimed at reducing mosquito populations. In the case of malaria, the most important control strategy globally has been Indoor Residual Spraying (IRS) of insecticides and use of Long-Lasting Insecticide Nets (LLIN's) [1]. However, widespread and increasing resistance to the most commonly used insecticides within the main vector mosquito species adapting to these indoor-targeted interventions has now resulted in clear indications of increasing malaria [1]. There are also indications that the aggressive deployment of IRS and LLINs has altered the feeding behaviour of mosquitoes from indoors to outdoors $[5,6]$. If this is the case, further reductions in the malaria incidence in Africa will require implementation of alternative vector control techniques [7, 8]. Therefore, targeting outdoor biting mosquitoes could have a significant impact in reducing malaria transmission. Topical repellents are used to prevent or 
at least reduce mosquito bites in typical outdoor settings. N,N-Diethyl-m-toluamide (DEET) is the most widely used topical repellent active [9-11]. The main problem with topical repellents is that their residual efficacy lasts for a few hours only [12]. This means that the repellent must be re-applied repeatedly and this makes it expensive for use in poor rural communities where malaria is most prevalent. Effective alternative formulations, including microencapsulated repellents that offer longer lasting residual efficacy are available but these tend to be prohibitively expensive for use in Africa [13].

The three most common malaria vectors in Africa, i.e. An. arabiensis, An. gambiae s.s. and An. funestus have a strong preference for feeding close to ground-level [14, 15]. In particular, for rural village inhabitants engaged in typical cooking and social activities, these mosquitoes overwhelmingly target the lower limbs, ankles and feet regardless of whether they are indoors or outdoors $[14,16]$. It has also been shown that under such conditions of standing or being seated, preventing access to these preferred areas of the body does not result in these malaria vector mosquitoes moving to other parts of the body, but that they will instead fly off to find other hosts with exposed lower limbs [14]. These findings suggest that the simple approach of protecting the selected body sites, i.e. ankles and feet, should help to reduce outdoor malaria transmission $[17,18]$.

Malaria is endemic in mostly poor and underprivileged areas of Africa [19-22]. Children in these areas often walk barefooted. This makes them vulnerable to malaria infection via lower limb mosquito bites. An intervention that can protect the lower limb region, the ankles and the feet, may therefore significantly reduce malaria transmission rates, particularly amongst children in Africa.

Long-lasting repellent fabrics have been developed. The most widely used insect repellents used for treating such fabrics are DEET and permethrin [23-25]. These repellents have been incorporated into the fabric materials using innovative techniques e.g. direct coating of permethrin onto fabric yarn [26] or microencapsulated repellents in fabric finishes [23, 27-29].

In this study, we report on the development of mosquito-repelling polymer fibres. The fibres were knitted into repellent fabrics intended for the protection of ankles and feet. The concept is based on bicomponent polymer filaments with the polymer in the core containing a volatile repellent added as a concentrate while a less permeable polymer is employed in the sheath layer. The development was conducted in two steps. In a first part principle parameters of core sheath fibre preparation was investigated using a piston spinning device. In a second part melt spinning was performed using universal spinning equipment with two extruders. The 
fibres were then knitted into repellent fabrics intended for the protection of ankles and feet. The durability of the repellence activity of such fabrics against cold washing as well as time was tested. The proposed technology may offer a route to cost-effective repellent textiles that may compete with other currently available repellent textile technologies. Such fabrics may facilitate affordable personal protection clothing items such as insect repellent socks or ankle covers.

\section{Materials and methods}

\subsection{Materials}

High density polyethylene (HDPE) was chosen as the sheath polymer. It was expected that it would feature a low permeability to the insect repellent because of its high crystallinity and the low solubility of DEET in this material. Borealis HDPE VL9500, a fibre grade HDPE suitable for bicomponent fibre extrusion, was chosen for the laboratory filament extrusion experiments. The melt flow index (MFI) of this material was $34 \mathrm{~g} / 10$ minutes at $190^{\circ} \mathrm{C} / 2.16 \mathrm{~kg}$. Owing to material availability issues, the pilot scale production trials utilised instead DOW grade $25055 \mathrm{E}$ (MFI $25 \mathrm{~g} / 10$ minutes at $190^{\circ} \mathrm{C} / 2.16 \mathrm{~kg}$; density $0.953 \mathrm{~g} \mathrm{~cm}^{-3}$ ) as the sheath polymer. According to the manufacturer, the latter materials has a very narrow molecular weight distribution. Based on the density, the crystallinity of this material is estimated at 70 wt.-\% while that of the Borealis grade VL9500 was 65 wt.-\% [30].

Preliminary tests showed that poly(ethylene-co-vinyl acetate) grades of high vinyl acetate content swell and absorb significant amounts of DEET when exposed to the liquid at elevated temperatures. Therefore Arkema EVA grade Evatane 2020 (MFI 17 - 23 g/10 minutes at $190^{\circ} \mathrm{C} / 2.16 \mathrm{~kg}$, density $940 \mathrm{~kg} \mathrm{~m}^{-3}$ and a VA content of 19-21\%) (EVA) was chosen for the core of the bicomponent filaments. DEET (stated purity of $97 \%$ purity, a density of $0.998 \mathrm{~g} \mathrm{~cm}^{-3}$, vapour pressure of $<1 \mathrm{~Pa}$ at $25^{\circ} \mathrm{C}$ and a normal boiling point of $288^{\circ} \mathrm{C}$ ) was supplied by SigmaAldrich and used as is. DEET has relatively very low volatility at room temperature. In a previous unpublished study, thermal stability investigations indicated that DEET is physically and chemically stable at melt fibre spinning conditions.

\subsection{DEET absorption by EVA}

The EVA pellets were loaded with DEET by facilitating swelling at elevated temperatures [31]. An excess quantity of DEET liquid added was sufficient to fully submerge the pellets in glass bottles. The containers were sealed and heated in a convection oven with the temperature set $81^{\circ} \mathrm{C}$ for $24 \mathrm{~h}$. The excess DEET was drained from the sampled bottles and 
methanol was used to rinse away any excess DEET still remaining on the surface of the polymer pellets. The pellets were then allowed to dry for a few minutes on paper towels before weighing. This process yielded swollen EVA pellets containing $40 \mathrm{wt}$.-\% DEET. This was established by thermogravimetric analysis (TGA) on a PerkinElmer TGA 4000 as follows: Samples in alumina pans were heated to high temperature at a rate of $10^{\circ} \mathrm{C} \mathrm{min}{ }^{-1}$ using inert nitrogen as the purge gas flowing at $50 \mathrm{~mL} \mathrm{~min}^{-1}$. EVA samples containing lower amounts of DEET were prepared by reducing the oven exposure time. Those were only used for the purpose of finding optimal fibre spinning conditions.

\subsection{Filament extrusion and fibre spinning}

Laboratory scale bi-component filament extrusion trials were conducted on the in-house designed-and-built piston melt spinning equipment at Leibniz Institute of Polymer Research Dresden e. V. (IPF Dresden e. V.). This system comprised two heated piston plunger pumps, one for the core component and the other for the sheath component. Drawdown was not possible with this setup but it was possible to vary the winding speed. Initial experiments aimed to establish the spinnability of the neat EVA in combination with the HDPE. Once suitable process conditions and material combinations were found, bi-component filament extrusion using DEET impregnated EVA pellets commenced, starting with low DEET levels and slowly increasing the concentration.

The pilot scale melt spinning trials were performed on a bicomponent extruder melt spinning machine. It consisted of two extruders. A single screw extruder was used for the core (Emil Blaschke model 74060-067) and a co-rotating twin screw extruder (Leistritz model MIC 18/GL-300) for the fibre sheath component. The melt spinning temperature was set at $160^{\circ} \mathrm{C}$. The melt generated by these two extruders was metered via separate gear pumps into the bicomponent die spinning head pack.

\subsection{Fibre and yarn characterization}

The filament or fibre samples were embedded in epoxy resin, cooled to liquid nitrogen temperatures after setting, and cut with a diamond knife. The specimens were gold coated before viewing on the SEM. The images were recorded with a Zeiss Ultra Plus field emission electron microscope at an acceleration voltage of $3 \mathrm{kV}$.

The concentration gradient of DEET in a laboratory-prepared filament was determined with a WITec alpha300R confocal Raman Imaging System as a function of filament depth. A 
$20 \times$ Zeiss objective was used. The Raman spectra were recorded using a $785 \mathrm{~nm} 75 \mathrm{~mW}$ laser. The integration time was $0.5 \mathrm{~s}$ and an average of 200 accumulations was recorded.

Tensile properties of the extruded filaments and pilot scale-produced yarn were determined on a $100 \mathrm{~N}$ Zwick/Roel Z0.5 universal testing machine at a draw speed of $200 \mathrm{~mm}$ $\min ^{-1}$.

\subsection{Knitting}

Knitting trials were performed at ITM, TU Dresden using a Shima Seiki flat knitting machine SWG091mini gauge E15. This machine type is ideally suited for the seamless manufacturing of net shape products. Slide needles and sinkers positioned very close to the needle enable a knitting process of complete net shape garments without the need of using a takedown system. To ensure a proper fabric structure six single bobbins were combined into a final yarn of 234 dtex.

The textile samples during this research are produced using flat knitting technology. The fabric formation in flat knitting takes place by feeding a yarn to individually programmable and movable needles. At the needles the yarns are formed into loops and several loops are interconnected to each other to form the fabric. Due to the loop structure knitted fabrics exhibit a high stretch ability, which make such perfectly suitable for the production of close to human skin worn products, e.g. underwear, hosiery or socks. Due to the high variety of binding combinations many possibilities of combination of the binding types and elements, knitted fabrics are also home textile articles and technical textiles for e.g. medical textiles, geotextiles such as knitted spacer fabrics, etc. are used.

Weft knitting is a very versatile textile manufacturing technique, which enables the direct production of 2D and 3D net-shape-structures without the need of additional assembly processes. Due to the flexibility of this technique, a variety of material combinations can be processed. In addition, a quick retooling of the machine is possible, so that a production of small batches up to lot size 1 is possible. 


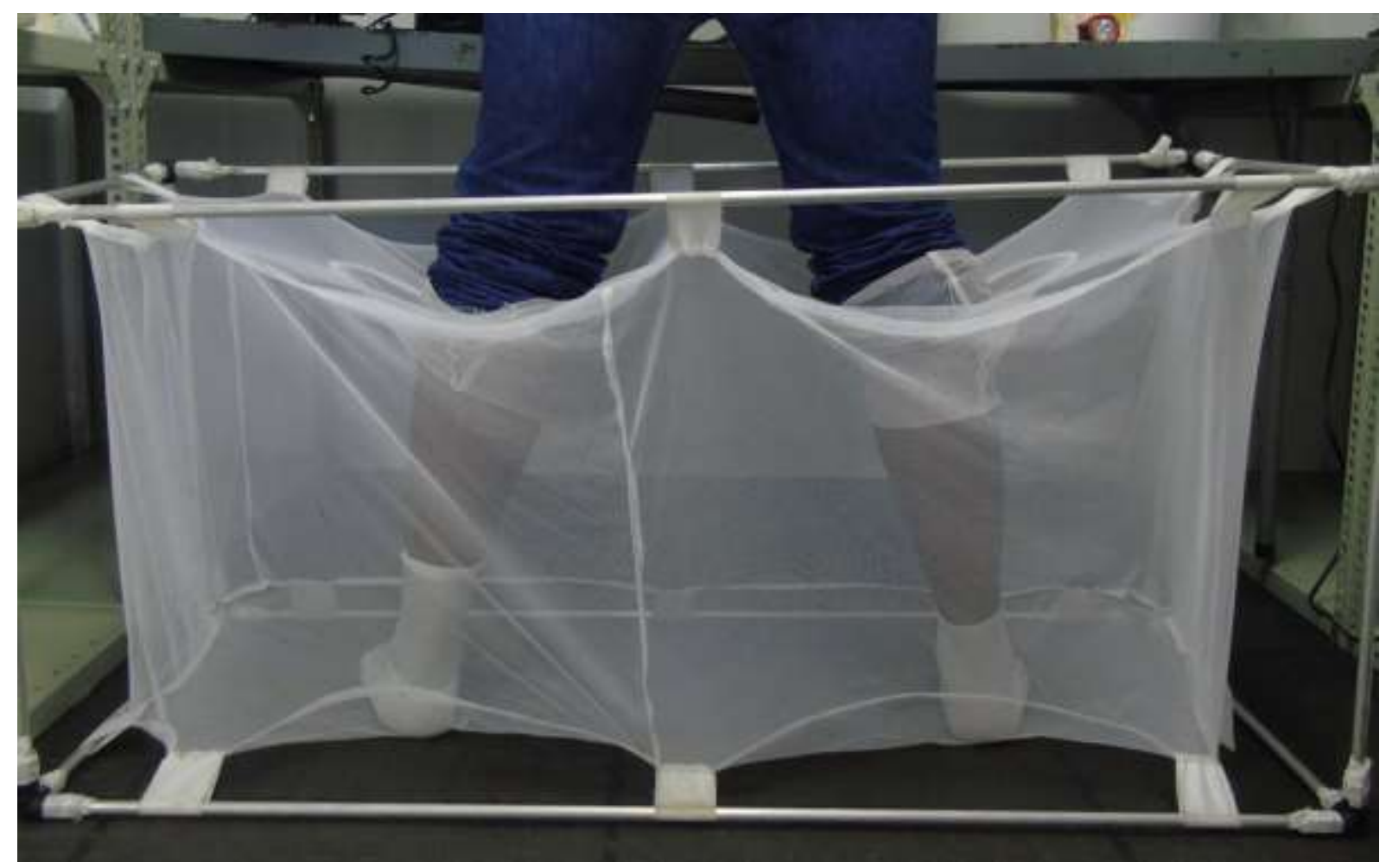

Figure 1: Illustration of foot-in-cage tests in progress.

\subsection{Repellence testing}

Repellent efficacy testing was performed using a foot-in-cage test. Ethical clearance for the study was obtained from the Faculty of Health Sciences ethics committee of the University of Pretoria (Protocol No. 82/2016). The tests for mosquito repellence efficacy were conducted under controlled insectary conditions of constant temperature $\left(25 \pm 2^{\circ} \mathrm{C}\right)$ and relative humidity $(75 \pm 5 \%)$. The caged mosquitoes were offered dual-choice opportunity for feeding at treated/untreated body parts of human volunteers [32, 33]. A colony of Anopheles arabiensis derived from stock material maintained by the South African National Institute for Communicable Diseases (NICD) was used. More than two hundred mixed-gender mosquitoes were placed in a large $(1.2 \times 0.6 \times 0.6 \mathrm{~m})$ netting cage. At the top it had two entry portals for the insertion of legs as shown in Figure 1. Every effort was made to ensure minimal disturbance of mosquitoes prior to each test. Mosquitoes used in these tests had not had access to blood-meals for $96 \mathrm{~h}$ to ensure that the female mosquitoes were starved and would readily try to bite and feed.

The foot-in-cage test was performed to determine how long the knitted socks retain residual effectiveness at ambient storage conditions. Three adult males were used as human volunteers to test the repellent socks. The treated sock was worn on the one foot and, as negative control, an untreated sock was worn on the other foot. Both feet were inserted into 
the cage for 5 minutes, at the end of which the number of mosquitoes that landed and probed or fed on the exposed feet were recorded. This bioassay test was performed once a month over a period of eight months. In-between testing, the treated sock was suspended in the air in a laboratory having a controlled temperature of $23^{\circ} \mathrm{C}$.

The protection $(p)$ afforded by the treated sock was calculated as the proportion of the number of mosquito-landings and/or probings on the treated leg $(T)$ in relation to the number of landings and/or probings on the control leg $(C)$ of the same individual:

$$
p=1-T / C
$$

\subsection{Wash resistance}

Wash resistance of the repellent fibres, knitted into gloves, was checked by an arm-in-cage test. Because there is no specific WHO protocol for testing the wash resistance of repellentimpregnated fabrics, a suitable evaluation method had to be established. It was decided to adapt the WHO wash testing protocol developed for insecticide impregnated nets [34]. It specifies methodologies for determining both the regeneration time and the wash resistance. The wash procedure for the gloves was as follows. The treated gloves were placed in a one litre beaker containing $0.50 \mathrm{~L}$ deionized water. One gram of soap was added and allowed to fully dissolve before washing commenced. At this point the $\mathrm{pH}$ was $10-11$. The beaker was placed in a waterbath at $30^{\circ} \mathrm{C}$ and shaken for 10 minutes at 155 movements per minute. The glove was removed, rinsed twice for 10 minutes in clean, deionized water under the same shaking conditions as above, dried at room temperature and stored at $30^{\circ} \mathrm{C}$ in the dark between washes.

For the arm-in-cage tests the rearing of mosquitoes was also done following a WHO recommended protocol [35]. The test mosquitoes were kept at a temperature of $27 \pm 2{ }^{\circ} \mathrm{C}$ and a relative humidity of $80 \pm 10 \%$. They were exposed to periods of $12 \mathrm{~h}$ light and $12 \mathrm{~h}$ darkness every day. A sugar solution (10\% glucose on cotton wool) was supplied for nourishment.

Testing was done using non blood-fed and host-seeking nulliparous female $A n$. arabiensis mosquitoes that were 5-8 days old. Handling of mosquitoes during testing was done via an aspirator apparatus. The repellent glove was worn on one hand of the investigator and a neat (untreated) glove was worn on the hand as a positive control. The hands were inserted in a cage with 250 female mosquitoes for 5 minutes. The number of mosquitoes that landed and probed or fed on the exposed hands were recorded. The degree of protection was calculated using equation (1). 
The regeneration time was determined by first conducting a repellent test on an unwashed glove sample to determine initial repellent effectiveness. This glove was subsequently washed and dried three times consecutively in a single day to deplete the repellent on the fabric surface. It was found, already on the following day, the glove provided $100 \%$ protective efficacy in an arm-in-cage tests. Therefore, one day, i.e. $24 \mathrm{~h}$, was taken as the regeneration time. Thereafter repellence tests were conducted after 0, 1, 3, 5, 10, 15, 20 and 25 washes. Clearly, the waiting period in between subsequent washes was longer that the regeneration time of one day as required. Each repellence test was performed just before the next wash.

\section{Results}

\subsection{Bicomponent monofilament extrusion}

Laboratory-scale filament extrusion experiments were conducted as a first step in the development of insect repellent filament fibres using a piston type spinning device. The objective was two-fold. First, suitable processing parameters and conditions for stable filament extrusion had to be established. Next the effect of the liquid-filled core polymer on the mechanical properties of the bicomponent filaments were determined. Details of that investigation are reported elsewhere [36]. In summary, it was found that the selected EVA grade was a suitable core polymer for bicomponent filaments. It was possible to reliably extrude bicomponent monofilaments at take up speeds approaching $800 \mathrm{~m} \mathrm{~min}^{-1}$ provided the final melt extrusion temperature was controlled at $160^{\circ} \mathrm{C}$. However, samples for analysis were extruded at an equal core and sheath throughput of ca. $0.50 \mathrm{~g} \mathrm{~min}^{-1}$ and a winding speed of 50 $\mathrm{m} \mathrm{min}^{-1}$. This allowed stable extrusion of filaments with a core diameter of ca. $90 \mu \mathrm{m}$ and a sheath thickness of ca. $20 \mu \mathrm{m}$, i.e. a total diameter of ca. $130 \mu \mathrm{m}$. DEET-free filaments as well as samples with ca. 10 wt.-\% and ca. 20 wt.-\% DEET were prepared for characterisation and analysis. The DEET content was checked by TGA mass loss measurements. Figure 2 shows mass loss curves for neat DEET and the laboratory fibre containing $20 \mathrm{wt}$. $\%$ DEET. Evaporation of the DEET was complete at $280^{\circ} \mathrm{C}$. The TGA curve for the fibre showed a plateau between $220^{\circ} \mathrm{C}$ and $290^{\circ} \mathrm{C}$ corresponding to the amount of DEET that was present. 


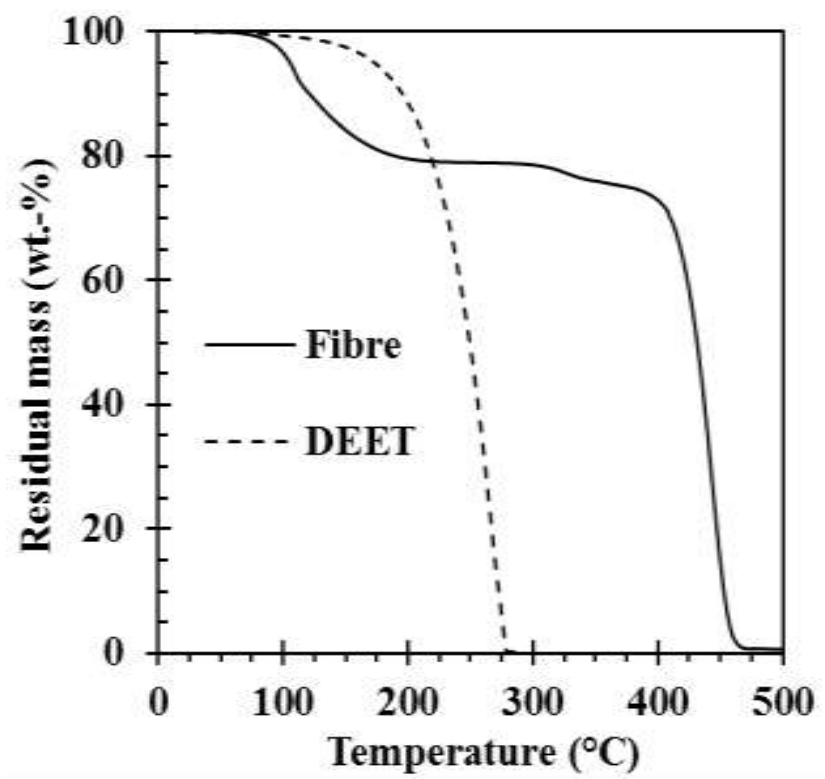

Figure 2: Thermogravimetric analysis of the evaporation of DEET in neat form and from the bicomponent fibre that contained ca. 20 wt.- $\%$ DEET. The scan rate was of $10^{\circ} \mathrm{C} \mathrm{min}^{-1}$ and the nitrogen purge gas flowed at $50 \mathrm{~mL}$ $\min ^{-1}$.

\subsection{Scanning Electron Microscopy of laboratory scale bicomponent filaments}

SEM cross-sectional micrographs of neat and DEET impregnated bicomponent filaments are shown in Figure 3. The micrographs show a well-defined core-sheath boundary. The shape of cross-sections deviated somewhat from circular and minor internal defects were observed especially in those filament in which DEET was present. 


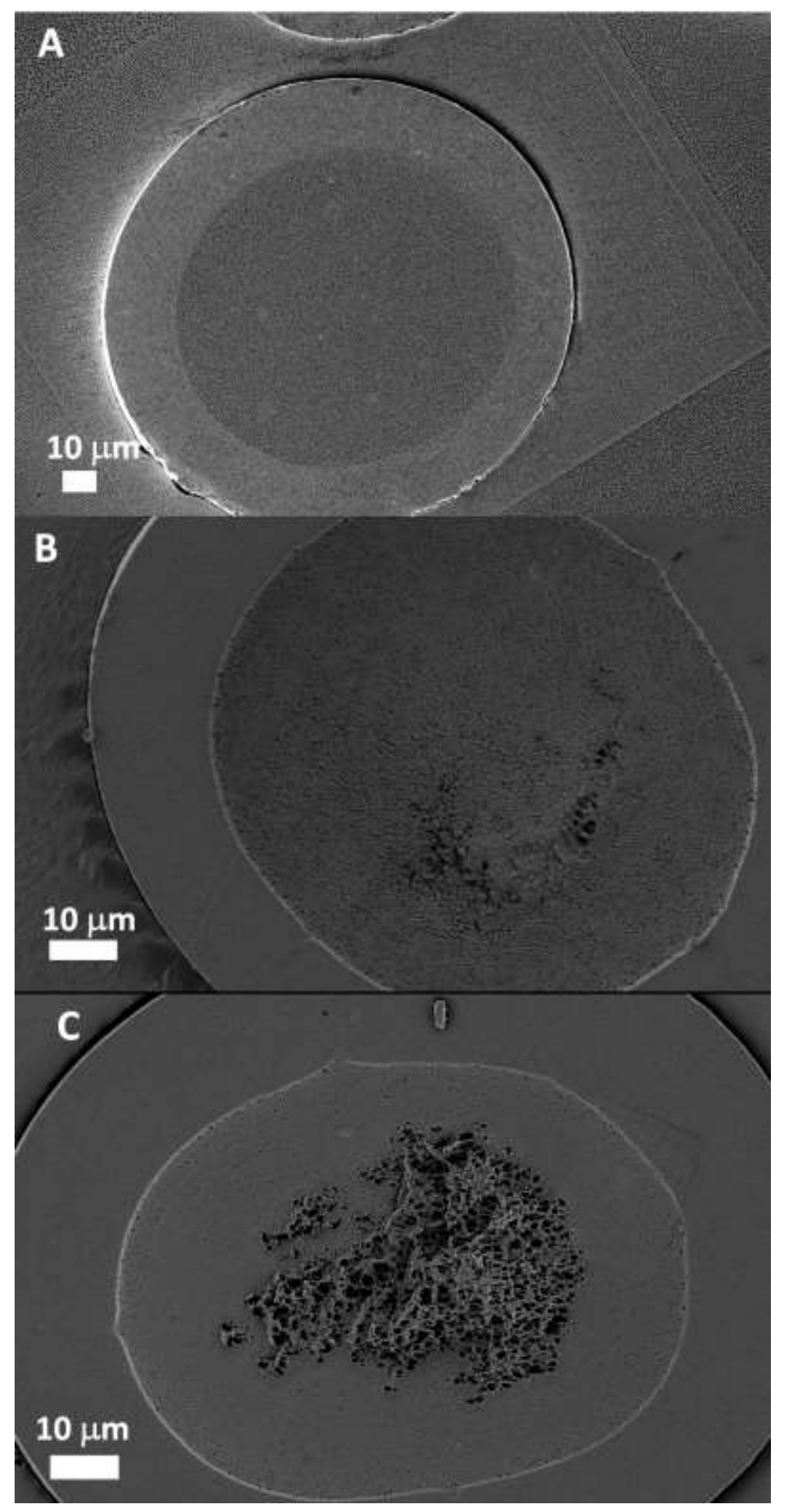

Figure 3: SEM micrographs of HDPE/EVA bicomponent filaments. They were extruded at an equal core and sheath throughput of $0.50 \mathrm{~g} \mathrm{~min}^{-1}$ and a draw down speed of $50 \mathrm{~m} \mathrm{~min}^{-1}$. DEET contents were: (A) No DEET, (B) ca. 10 wt.-\% DEET, and (C) ca. 20 wt.-\% DEET.

A closer inspection of the filament impregnated with ca. 20 wt.-\% DEET (Figure 3 C) reveals porous structures reminiscent of a microporous polymer matrix. It is very likely that 
much of the DEET repellent was actually present in these pores. Even in this filament, there is a clear boundary between the EVA and HDPE phases. Retention of the integrity of the polyethylene sheath layer is important if it is to act as a permeable membrane barrier for controlling the active release rate.
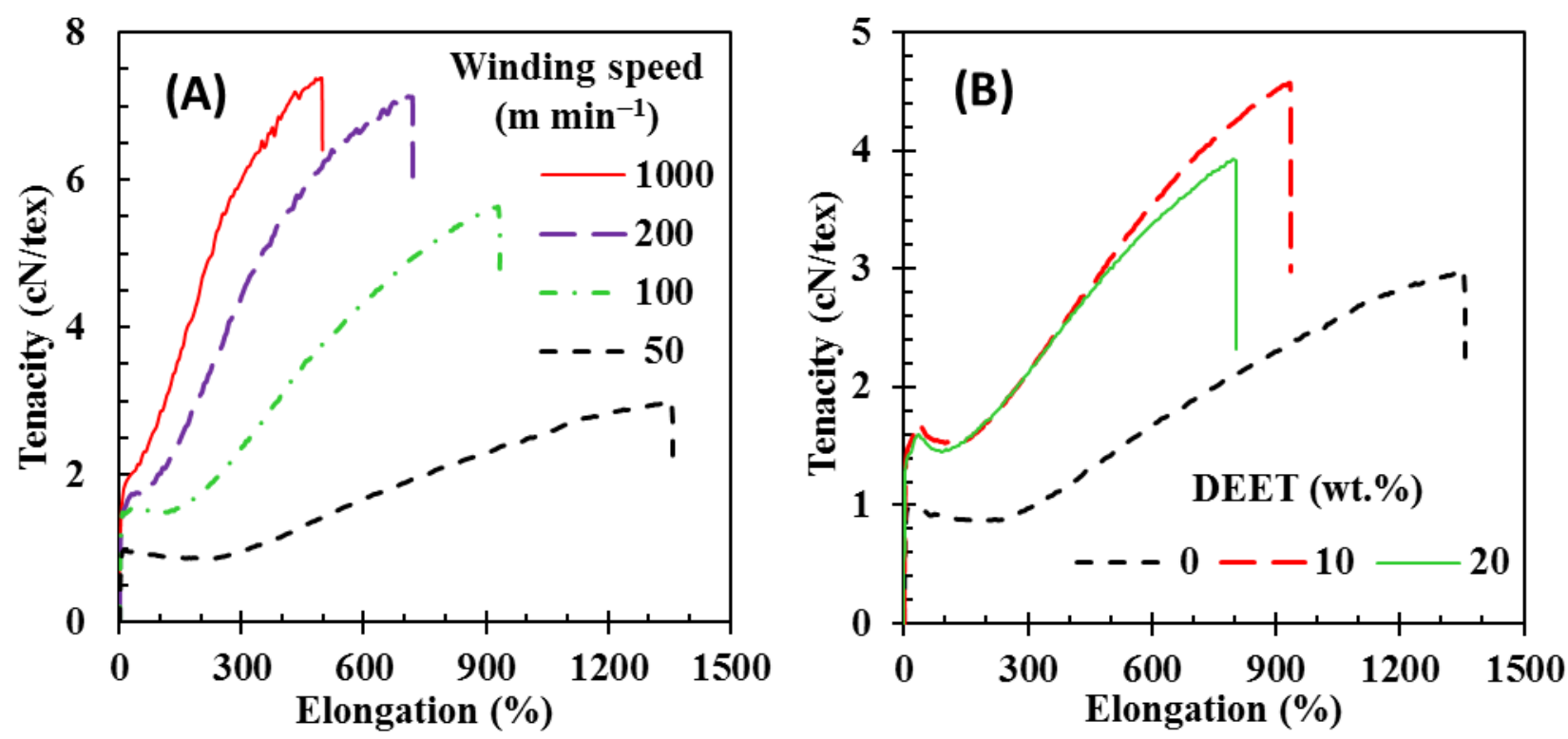

Figure 4: (A) Representative stress-strain curves showing the effect of take up speed at constant polymer melt throughput on neat bicomponent filaments. (B) The effect of DEET content on tenacity for fibres spun at a winding speed in is $50 \mathrm{~m} \mathrm{~min}^{-1}$.

\subsection{Filament mechanical properties}

Representative stress-strain curves for the bicomponent filaments extruded at a total throughput of ca. $1 \mathrm{~g} \mathrm{~min}^{-1}$ at $160{ }^{\circ} \mathrm{C}$ are shown in Figure 4. Figure 4 (A) shows the effect of varying the winding speed on the properties of DEET-free filaments. Mechanically, the filament was quite weak. In all plots, plastic deformation starts at a very low extension ratio. Considerable progressive strain hardening is observed over a wide extension range before the filament breaks. The tenacity increased with increase in the winding speed. This is attributable to increased orientation of the polymer chains in the fibre direction. Interestingly, the filaments show very high elongations to break which suggests that significantly better mechanical properties would have been possible had the filaments been drawn. This is partly due to the presence of EVA and is a desirable characteristic for a typical knitting process [37].

Figure 4(B) shows representative plots of tenacity against strain for filaments containing different DEET concentrations. The winding speed was kept constant at $50 \mathrm{~m}$ $\mathrm{min}^{-1}$. Surprisingly, the presence of DEET increased the tenacity of the filaments. At this point one can only speculate about possible reasons for this strange and unexpected 
observation. More fundamental studies will be required to determine the mechanisms responsible for this effect.
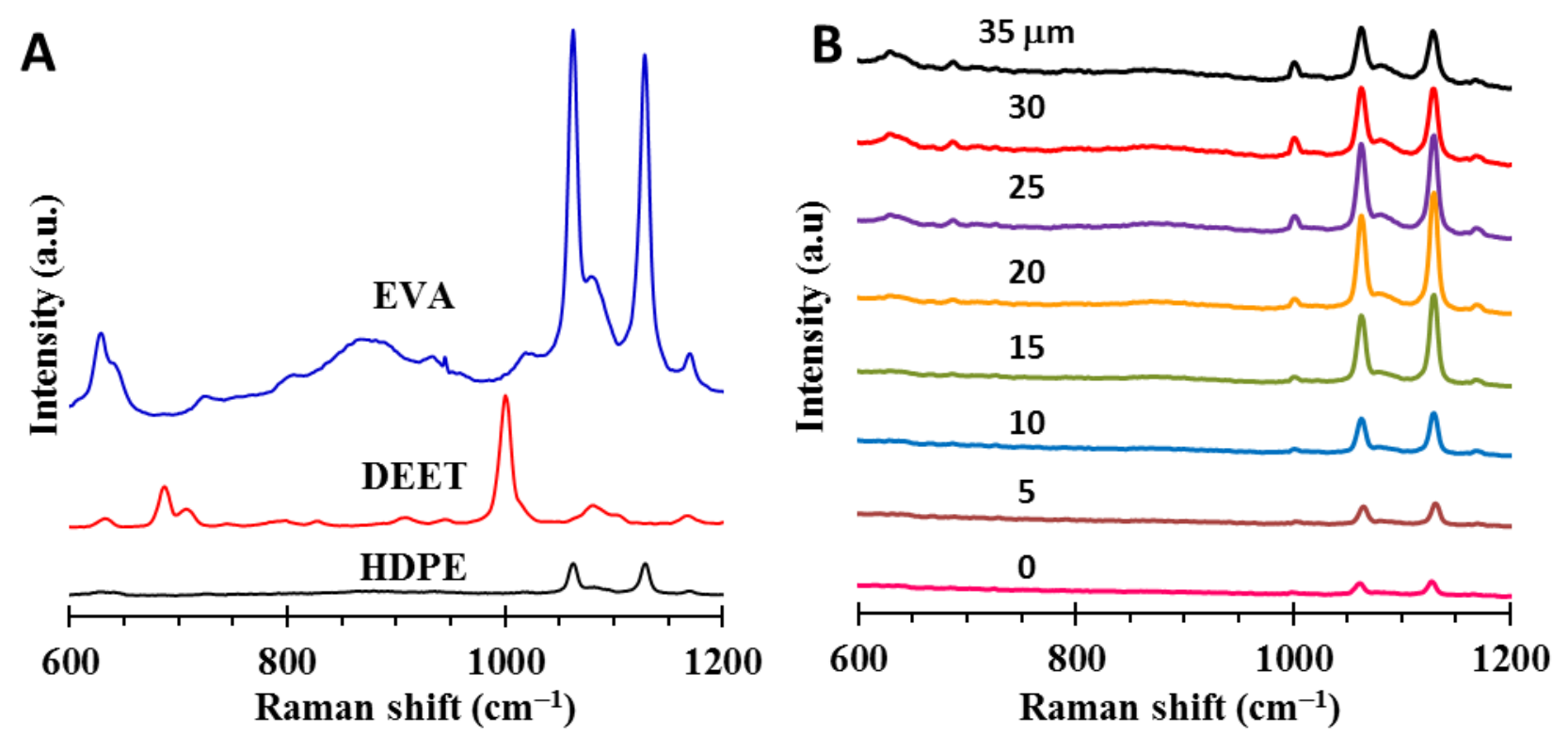

Figure 5: Raman spectra of a bicomponent filament that contained $20 \mathrm{wt} .-\%$ DEET based on the total mass of core polymer and repellent. (A) Spectra for DEET compared to those for EVA and HDPE. (B) Confocal Raman spectra recorded at different positions inside the fibre with the distance from the surface indicated in units of $\mu \mathrm{m}$.

\subsection{Raman study of bicomponent filaments}

The process of bicomponent filament spinning involves the drawing and cooling of the polymer melt. The stretching aligns the polymer chains in the axial direction increasing the tenacity of the filament. As the material cools, the polymer phases crystallize reducing the amount of amorphous matrix available for dissolving the repellent active. DEET is a spatial repellent. It has to be available as a vapour in the immediate atmosphere surrounding the filament in sufficient quantities to effectively repel mosquitoes. The repellent in the vapour phase must constantly be replenished by the liquid repellent that has diffused to the surface of the filament. This implies diffusion of the liquid repellent from the core part of the filament (EVA phase) to the surface via the HDPE sheath. Consequently, there should be a concentration gradient in the radial fibre direction. Furthermore, the gradient in the sheath layer should be steeper than in the core as it is expected to function as a rate-controlling barrier layer [38]. The confocal Raman study, on the laboratory prepared filaments, was used to test this concentration profile hypothesis. The Raman laser beam was focused within the filament at different depths to determine the respective DEET concentrations. Figure 5(A) shows Raman spectra recorded for neat samples of DEET, EVA and HDPE. Figure 5(B) 
shows spectra obtained at different depths for the filament containing ca. 20 wt.-\% DEET. The DEET spectrum features a characteristic band located at $1000 \mathrm{~cm}^{-1}$ that is not present in the spectra of the two polymers. The concentration of DEET at different positions in the filament should be proportional to the area under this peak. Figure 6 shows plots of the corresponding area of this DEET absorption band. The peak areas were evaluated using Origin Pro ${ }^{\circledR}$ software. This absorption band gradually increases in in intensity and in area as the laser focus is set deeper inside the filament. This confirms that there is indeed a concentration gradient in the radial fibre direction, particularly across the sheath layer. This indicates controlled release of DEET from the bicomponent filament.

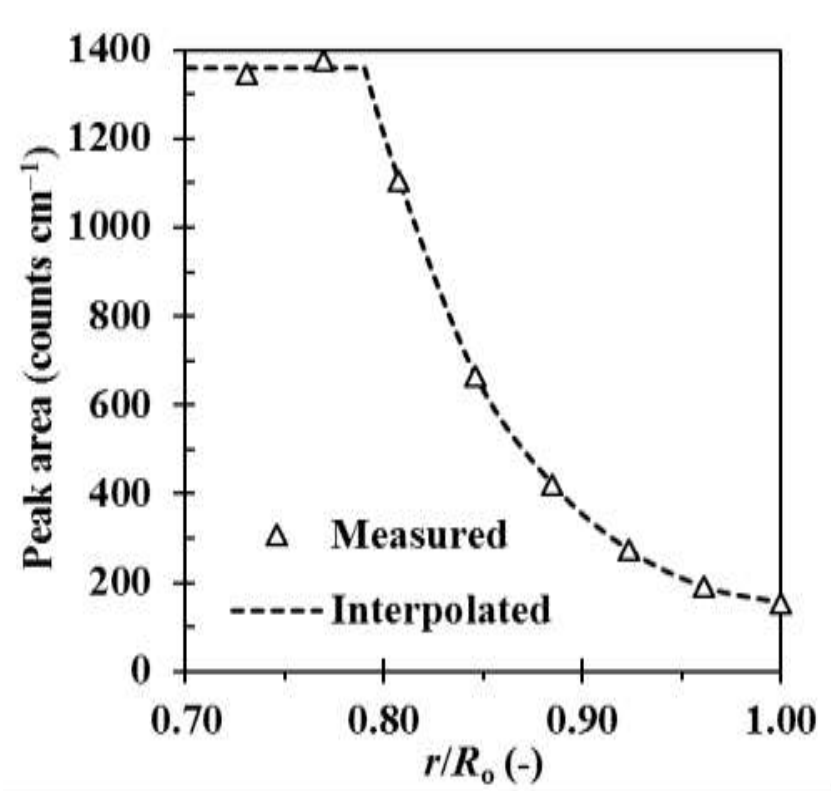

Figure 6: Variation of DEET concentration with normalized radial position inside a filament that contained ca. 20 wt. $-\%$ of the active. $R_{\mathrm{o}}$ is the radius of the bicomponent fibre and $r$ is the radial distance from the centre. The concentration is expressed in terms of the area under the DEET absorption band located at $1000 \mathrm{~cm}^{-1}$.

\subsection{Yarn spinning}

The final bicomponent yarn was produced using an 11-holes spinneret. Stable pilot scale spinning was achieved when the yarn, used for knitting, was produced at $3000 \mathrm{~m} \mathrm{~min}^{-1}$. The total core and sheath throughputs were set at $7 \mathrm{~g} \mathrm{~min}^{-1}$ and $5 \mathrm{~g} \mathrm{~min}^{-1}$ and this resulted in yarn with titre ranging from 39 - 45 dtex. The tensile strength, of the melt spun fibres, was further improved by increasing the draw ratio up to 3 .

\subsection{Scanning electron microscopy of laboratory scale bicomponent filaments}

Figure 7 shows SEM micrographs of cross-sections of neat and DEET impregnated filaments obtained by pilot-scale bicomponent multi-filament yarn spinning. The neat yarn was spun 
using approximately equal parts of HDPE and EVA. However in the presence of DEET, the HDPE phase contribution had to be increased to ca. $60 \mathrm{wt} . \%$ HDPE in order to attain stable yarn spinning conditions. This resulted in an increase in the sheath thickness.

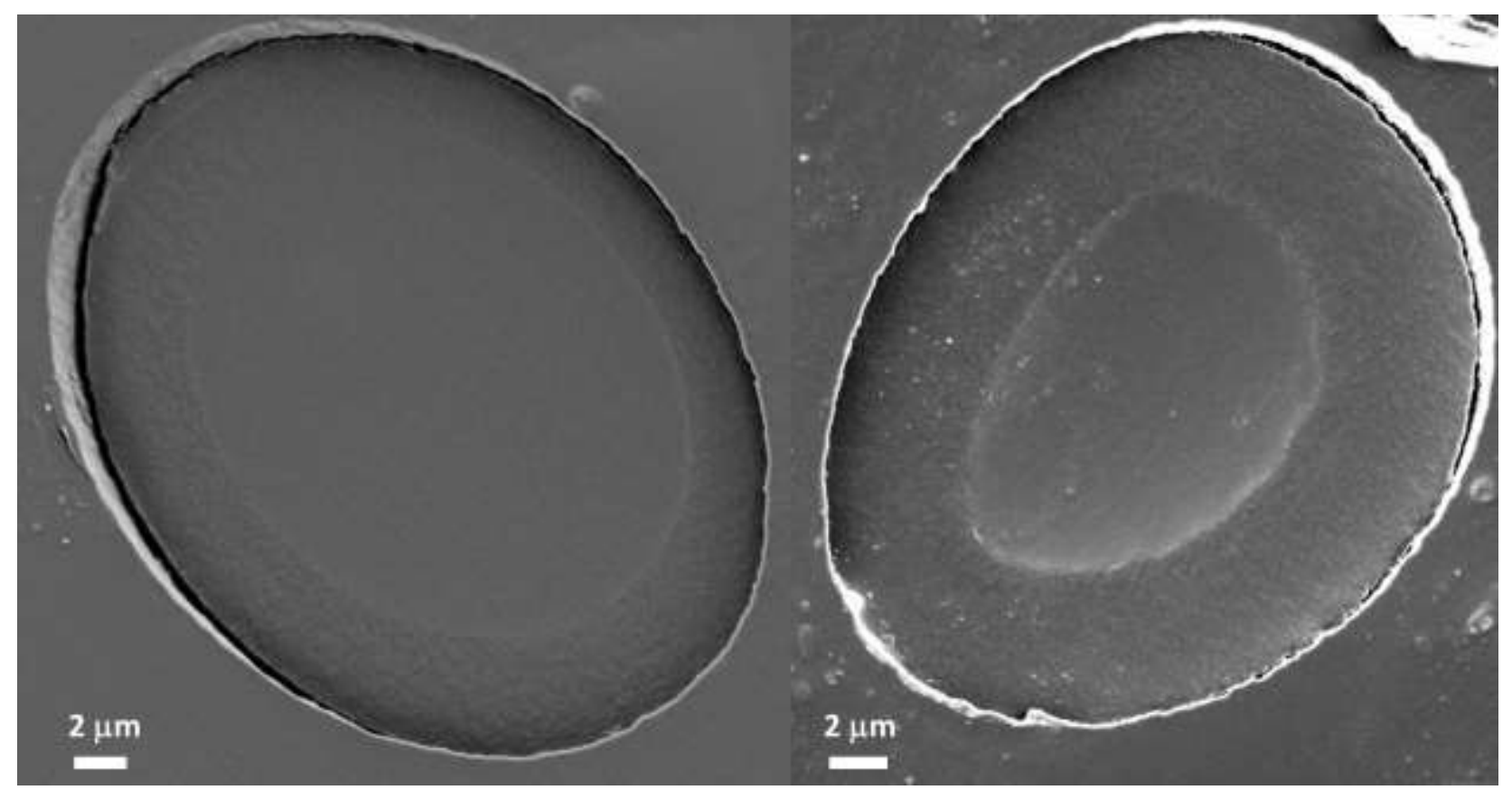

Figure 7: SEM micrographs of bicomponent yarn filament containing (A) no DEET and (B) 20 wt.-\% DEET.

\subsection{Yarn mechanical properties}

The knitting process requires yarn with a sufficient strength. It is important to produce a yarn with a reasonably high tenacity in order to minimise breakages during the knitting process.

Figure 8 shows tensile test results for yarn containing $17 \mathrm{wt} .-\%$ DEET spun at $3000 \mathrm{~m} \mathrm{~min}^{-1}$. As expected, the tenacity increased with the draw ratio. The final yarn was produced at a draw ratio of three. 

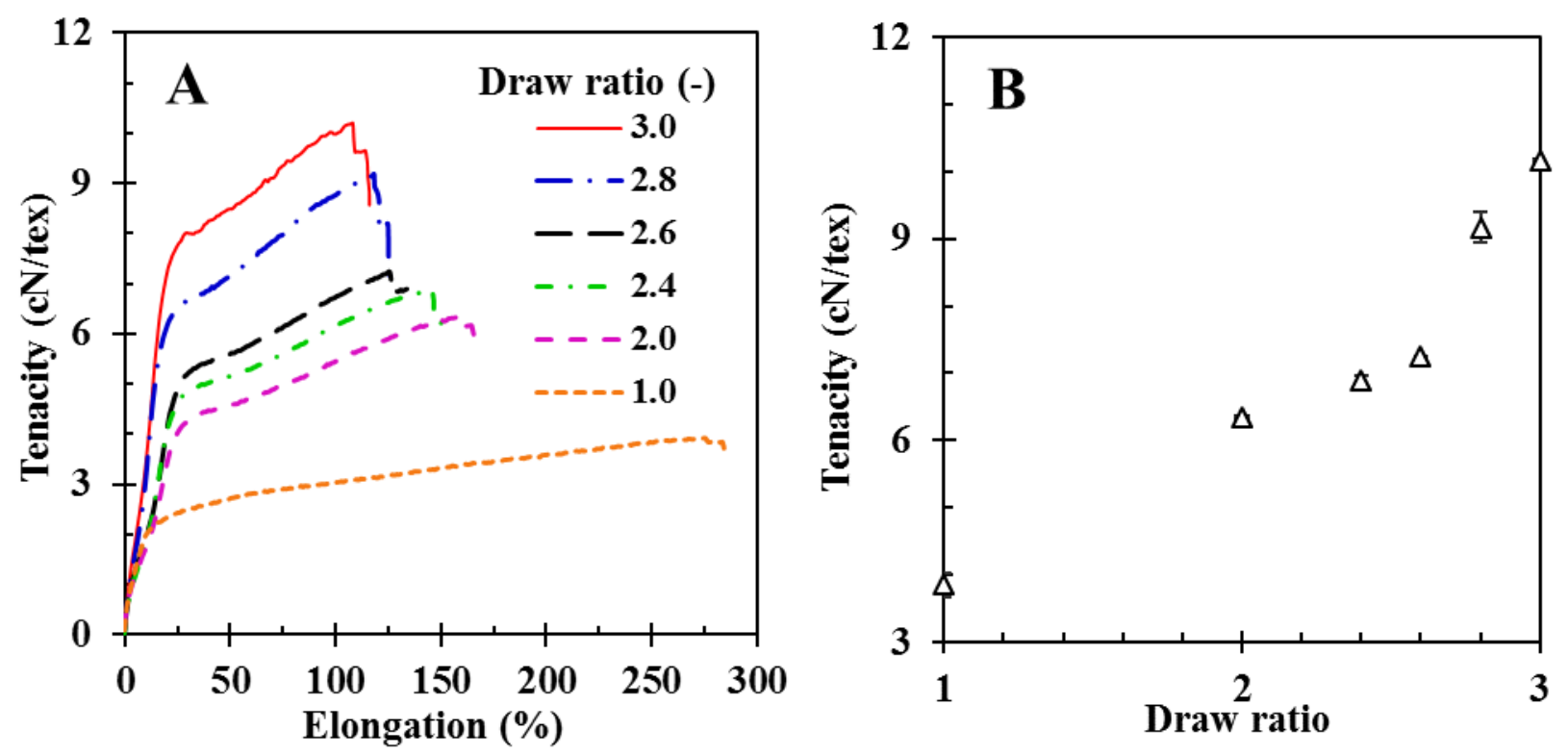

Figure 8: Mechanical properties for 11-filament bicomponent yarn impregnated with 17 wt.-\% DEET and spun at $3000 \mathrm{~m} \mathrm{~min}^{-1}$. (A) Typical tenacity vs. elongation curves. (B) Plot of tenacity against elongation.

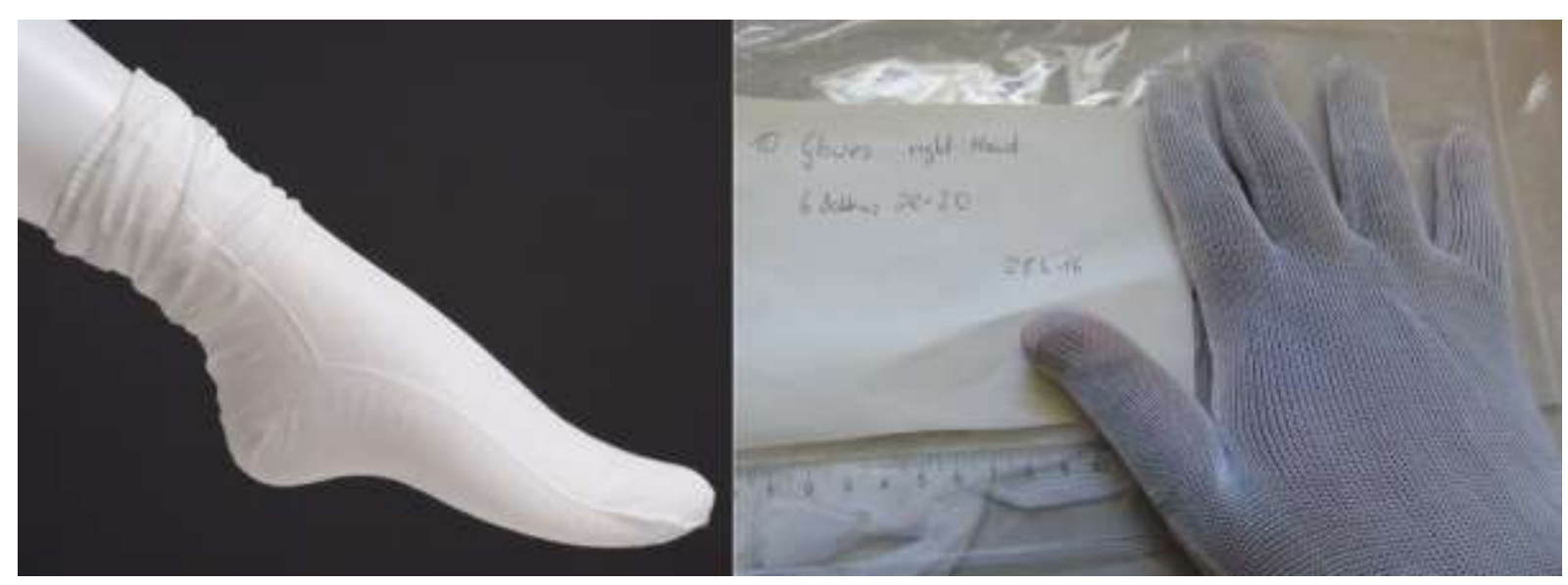

Figure 9: Images of sample repellent sock and glove

\subsection{Textile knitting}

The final 11-filament bicomponent yarn contained 17 wt.-\% DEET and was spun at 3000 $\mathrm{m} \min ^{-1}$ using a draw ratio of 3 . To ensure a proper fabric structure, six single bobbins were combined into a final 234 dtex yarn. Gloves and socks were knitted from the yarn produced. Figure 9 shows an example of the knitted sock. Due to a smooth surface of the yarn, difficulties were experienced with setting a proper yarn tension when using friction-based electronic yarn feeders. Therefore, a mechanical system based on springs and yarn brakes was used to set the yarn tension. Special features of the machine such as the yarn insertion system enabled a start of the knitting on available empty needle across the needle bed. This system also enabled the individual manufacturing of every finger of the glove. Using the loop transfer 
technique, the separate glove fingers were automatically combined into one knitted glove. The yarns generally exhibited very good textile processing properties. The knitting trials were performed without any interruption being caused by a yarn breakage or other yarn related issues.

\subsection{Repellent testing}

The foot-in-cage repellent test results are shown in Figure 10. The sock showed impressive residual effectiveness with more than $90 \%$ protective efficacy after 20 weeks kept at laboratory conditions. The sock even exhibited partial repellent effectiveness up to week 33. A significant reduction in mosquito bites reduces the odds of successful malaria parasite transmission to humans.

Figure 11 shows the results of wash resistance studies conducted on sample gloves. The fabric retained $100 \%$ repellent effectiveness even after 20 cold washes. The fabric showed partial repellent effectiveness after the $25^{\text {th }}$ wash. It is the considered opinion that of the authors that this is a good result

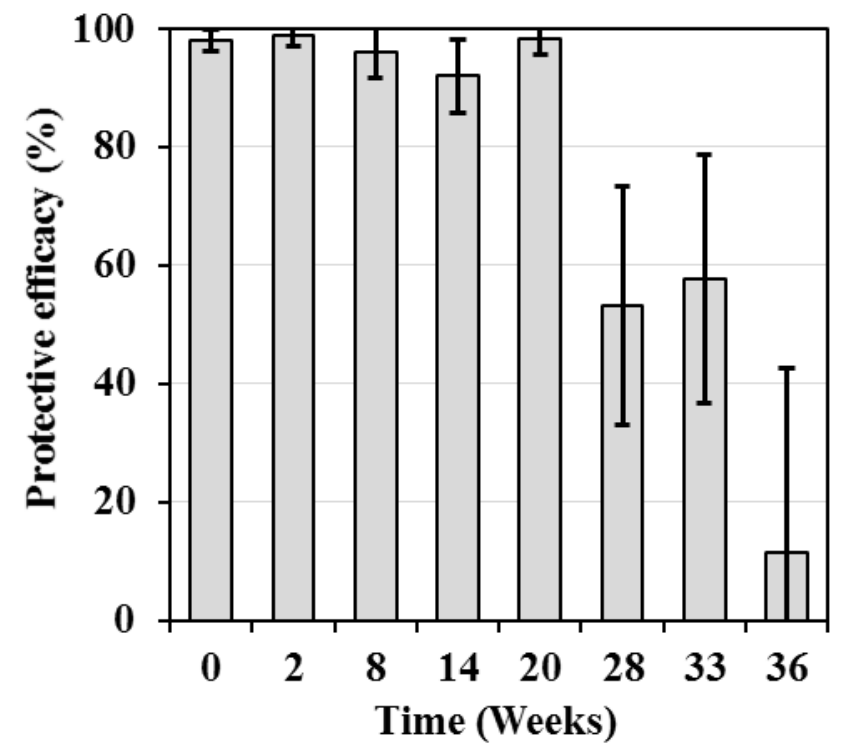

Figure 10: Bar plot of foot-in-cage repellence tests. Error bars represent 95\% confidence intervals. 


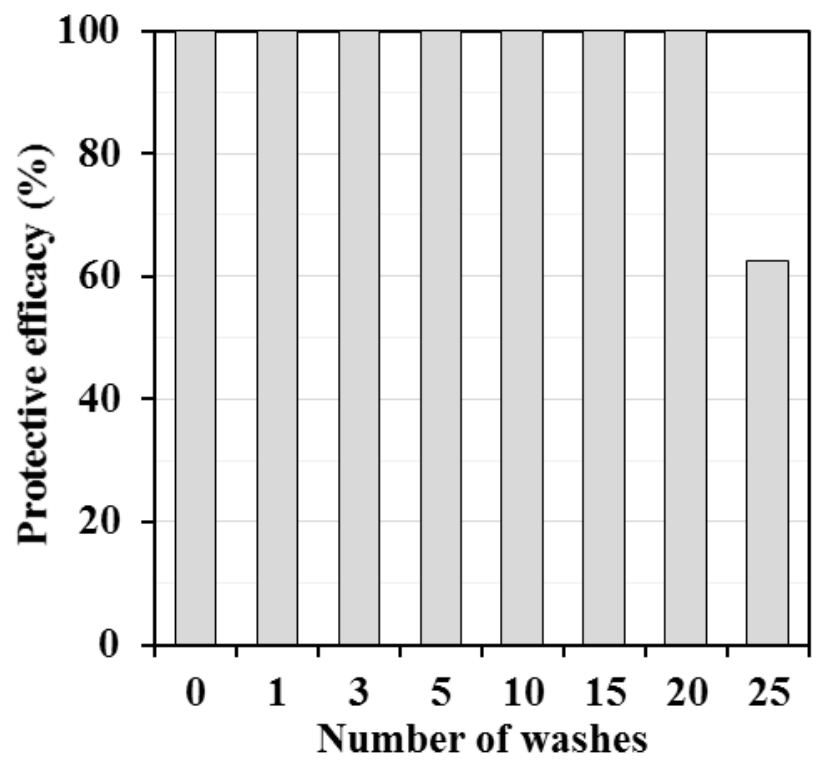

Figure 11: Bar plot of arm-in-cage wash resistance tests

\section{Discussion}

The development of the bicomponent filament was done using HDPE and EVA. These polymers are relatively inexpensive and this will could make the final product affordable. DEET was used as the volatile active. It was selected because it is the most widely used and most effective repellent on the market [12]. However, there have been concerns about the toxicity of this repellent particularly towards young children [39]. Future studies should consider incorporating alternatives to DEET into the bicomponent filaments. IR3535, ethyl anthranilate and picaridin are effective synthetic alternatives. $p$-Menthane-3,8-diol may be considered as a potential natural repellent alternative.

\section{Conclusions and recommendations}

Increasing the residual effectiveness of repellents could make a significant impact in reducing outdoor malaria transmission. The work presented here has proven the concept of trapping a volatile repellent in a bicomponent filament and subsequent slow release as a viable and effective technique with the potential to reduce outdoor mosquito bites for up to 33 weeks or at least 20 cold washes. These filaments can be manufactured cost effectively using a simple melt extrusion and drawing process. This technology may offer an affordable textile based long lasting repellent intervention that can be deployed in disadvantaged communities where malaria is mostly prevalent. Products manufactured from these bicomponent filaments should be tested in large scale field trials to evaluate their impact on reducing malaria transmission particularly in endemic communities. 


\section{Acknowledgements}

The authors thank Mathias Häschel and Norbert Smolka for technical assistance during the melt spinning trials. Thanks are also due to Dr Joel Mouatcho, Cyril Ndonyane and Allison Gilbert for assistance in performing repellent testing. Financial support from the Institute of Applied Materials, the National Research Foundation (NRF), the Technology Innovation Agency (TIA) and the Leibniz Institute of Polymer Research (IPF) is also acknowledged.

\section{References}

[1] WHO, World malaria report 2017, Geneva, 2017.

[2] S. Wynd, D.N. Durrheim, J. Carron, B. Selve, J.P. Chaine, P.A. Leggat, W. Melrose, Socio-cultural insights and lymphatic filariasis control - Lessons from the Pacific, Filaria Journal, 6 (2007).

[3] L. Braack, A.P. Gouveia De Almeida, A.J. Cornel, R. Swanepoel, C. De Jager, Mosquito-borne arboviruses of African origin: Review of key viruses and vectors, Parasites and Vectors, 11 (2018).

[4] S.C. Weaver, W.K. Reisen, Present and future arboviral threats, Antiviral Research, 85 (2010) 328-345.

[5] N. Moiroux, M.B. Gomez, C. Pennetier, E. Elanga, A. Djènontin, F. Chandre, I. Djègbé, H. Guis, V. Corbel, Changes in anopheles funestus biting behavior following universal coverage of long-lasting insecticidal nets in benin, Journal of Infectious Diseases, 206 (2012) 1622-1629.

[6] T.L. Russell, N.J. Govella, S. Azizi, C.J. Drakeley, S.P. Kachur, G.F. Killeen, Increased proportions of outdoor feeding among residual malaria vector populations following increased use of insecticide-treated nets in rural Tanzania, Malaria Journal, 10 (2011).

[7] R.W. Snow, K. Marsh, Malaria in Africa: Progress and prospects in the decade since the Abuja Declaration, The Lancet, 376 (2010) 137-139.

[8] M. Tanner, D. De Savigny, Malaria eradication back on the table, Bulletin of the World Health Organization, 86 (2008) 82.

[9] M. Debboun, S. Frances, D. Strickman, Insect repellents, Principles, methods, and uses, Taylor \& Francis group, Boca Raton, 2007, pp. 465.

[10] C. Costantini, A. Badolo, E. Ilboudo-Sanogo, Field evaluation of the efficacy and persistence of insect repellents DEET, IR3535, and KBR 3023 against Anopheles gambiae complex and other Afrotropical vector mosquitoes, Transactions of the Royal Society of Tropical Medicine and Hygiene, 98 (2004) 644-652.

[11] T.W. Walker, L.L. Robert, R.A. Copeland, A.K. Githeko, R.A. Wirtz, J.I. Githure, T.A. Klein, Field evaluation of arthropod repellents, deet and a piperidine compound, AI3-37220, against Anopheles funestus and Anopheles arabiensis in western Kenya, Journal of the American Mosquito Control Association, 12 (1996) 172-176.

[12] E. Lupi, C. Hatz, P. Schlagenhauf, The efficacy of repellents against Aedes, Anopheles, Culex and Ixodes spp. - A literature review, Travel Medicine and Infectious Disease, 11 (2013) 374-411.

[13] J. Kitau, R. Oxborough, J. Matowo, F. Mosha, S.M. Magesa, M. Rowland, Indoor residual spraying with microencapsulated DEET repellent ( $\mathrm{N}, \mathrm{N}$-diethyl-m-toluamide) for control of Anopheles arabiensis and Culex quinquefasciatus, Parasites and Vectors, 7 (2014).

[14] L. Braack, R. Hunt, L.L. Koekemoer, A. Gericke, G. Munhenga, A.D. Haddow, P. Becker, M. Okia, I. Kimera, M. Coetzee, Biting behaviour of African malaria vectors:1. Where do the main vector species bite on the human body?, Parasites and Vectors, 8 (2015).

[15] J.D. Charlwood, J. Pinto, P.R. Ferrara, C.A. Sousa, C. Ferreira, V. Gil, V.E. do Rosário, Raised houses reduce mosquito bites, Malaria Journal, 2 (2003) 45.

[16] L.E.O. Braack, M. Coetzee, R.H. Hunt, H. Biggs, A. Cornel, A. Gericke, Biting pattern and host-seeking behavior of Anopheles arabiensis (Diptera: Culicidae) in northeastern South Africa, Journal of Medical Entomology, 31 (1994) 333-339.

[17] D.N. Durrheim, J.M. Govere, Malaria outbreak control in an African village by community application of 'deet' mosquito repellent to ankles and feet, Medical and Veterinary Entomology, 16 (2002) 112-115.

[18] J. Govere, L.E.O. Braack, D.N. Durrheim, R.H. Hunt, M. Coetzee, Repellent effects on Anopheles arabiensis biting humans in Kruger Park, South Africa, Medical and Veterinary Entomology, 15 (2001) 287-292.

[19] R.I. Chima, C.A. Goodman, A. Mills, The economic impact of malaria in Africa: A critical review of the evidence, Health Policy, 63 (2003) 17-36. 
[20] C. Goodman, K. Hanson, A. Mills, V. Wiseman, E. Worrall, The Economics Of Malaria And Its Control, Paper for the WHO/TDR Scientific Working Group on Malaria, World Health Organisation, Geneva, 2003.

[21] J. Sachs, P. Malaney, The economic and social burden of malaria, Nature, 415 (2002) 680-685.

[22] J.L. Gallup, J.D. Sachs, The economic burden of malaria, American Journal of Tropical Medicine and Hygiene, 64 (2001) 85-96.

[23] B. Fei, J.H. Xin, N, N-diethyl-m-toluamide-containing microcapsules for bio-cloth finishing, American Journal of Tropical Medicine and Hygiene, 77 (2007) 52-57.

[24] X. Deparis, B. Frere, M. Lamizana, R. N'Guessan, F. Leroux, P. Lefevre, L. Finot, J.M. Hougard, P. Carnevale, P. Gillet, D. Baudon, Efficacy of permethrin-treated uniforms in combination with DEET topical repellent for protection of French military troops in Côte d'Ivoire, Journal of Medical Entomology, 41 (2004) 914-921. [25] T.H. Lillie, C.E. Schreck, A.J. Rahe, Effectiveness of personal protection against mosquitoes in Alaska, Journal of Medical Entomology, 25 (1988) 475-478.

[26] M. Faulde, W. Uedelhoven, A new clothing impregnation method for personal protection against ticks and biting insects, International Journal of Medical Microbiology, 296 (2006) 225-229.

[27] M. Sumithra, N. Vasugi Raja, Mosquito repellency finishes in blended denim fabrics, International Journal of Pharmacy and Life Sciences 3(2012) 1614-1616.

[28] R. Anitha, T. Ramachandran, R. Rajendran, M. Mahalakshmi, Microencapsulation of lemon grass oil for mosquito repellent finishes in polyester textiles Elixir International Journal, 40 (2011) 5196-5200.

[29] R. N'Guessan, B.G.J. Knols, C. Pennetier, M. Rowland, DEET microencapsulation: a slow-release formulation enhancing the residual efficacy of bed nets against malaria vectors, Transactions of the Royal Society of Tropical Medicine and Hygiene, 102 (2008) 259-262.

[30] B. Kutlu, J. Meinl, A. Leuteritz, H. Brünig, G. Heinrich, Melt-spinning of LDH/HDPE nanocomposites, Polymer, 54 (2013) 5712-5718.

[31] A. Senuma, SWELLING MEASUREMENT OF SOME ETHYLENE COPOLYMERS CROSSLINKED BY DICUMYL PEROXIDE, J Polym Sci Part A-1 Polym Chem, 12 (1974) 2631-2638.

[32] WHO, Guidelines for efficacy testing of mosquito repellents for human skin, World Health Organisation, Geneva, 2009.

[33] D.R. Barnard, R.D. Xue, Laboratory evaluation of mosquito repellents against Aedes albopictus, Culex nigripalpus, and Ochlerotatus triseriatus (Diptera: Culicidae), Journal of Medical Entomology, 41 (2004) 726730.

[34] WHO, Guidelines for for laboratory and field-testing of long-lasting insecticidal nets, World Health Organisation, Geneva, 2013.

[35] WHO, Guidelines for efficacy testing of spatial repellents, in: W.H. Organisation (Ed.) Control of neglected tropical diseases, WHO pesticide evaluation scheme, World Health Organisation, Geneva, 2009.

[36] S. Sibanda, A. Leuteritz, H. Brünig, W. Focke, Development of polyolefin bicomponent filaments as controlled release devices of volatile repellents for use in malaria vector control Europe Africa Regional Conference of the International Polymer Processing Society (PPS), Dresden, Germany, 2017.

[37] C. Lawrence, Fundamentals of spun yarn technology, CRC Press, Florida, 2005.

[38] W. Beek, K. Mutzall, J. van Heuven, Transport Phenomena, Wiley \& Sons, Chichester, 1999.

[39] G. Koren, D. Matsui, B. Bailey, DEET-based insect repellents: Safety implications for children and pregnant and lactating women, CMAJ, 169 (2003) 209-212. 